\title{
Preparation and Application of a Xylan-Based Antibacterial Additive Agent against Escherichia Coli Bacteria
}

Guibin Xu a , Tao Song ${ }^{\text {a, }}$, Bei He ${ }^{\text {a }}$, Minmin Chang a , Junli Ren ${ }^{\text {b, * }}$

${ }^{\text {a }}$ State Key Laboratory of Pulp and Paper Engineering, South China University of Technology,

Guangzhou, 510640, China

${ }^{\mathrm{b}}$ School of Light Industry and Engineering, South China University of Technology, Guangzhou, 510340, China

Correspondence to: T. Song (E-mail: songt@scut.edu.cn) and J. Ren (E-mail: renjunli@scut.edu.cn)

Abstract: In this work, a xylan-based antimicrobial additive agent was prepared and aimed for uses in paper products against Escherichia coli bacteria. The derived Cationic-Xylan-grafted-PHGH (CX-g-PHGH) was successfully synthesized by graft copolymerization of cationic-xylan with guanidine polymer (PHGH) using ceric ammonium nitrate as initiator. The obtained CX-g-PHGH had maximum PHGH grafting ratio of $18.45 \%$ and efficiency of $58.45 \%$, and showed good viscosity and thermal stability. Furthermore, the paper samples prepared in this work were reinforced obviously with the addition of CX-g-PHGH by improved mechanical properties. Compared to the reference paper without any of the xylan-derivatives, the index of tensile, tear, burst and folding endurance of the paper had increases up to $20.07 \%$, $25.31 \%, 30.20 \%$ and $77.78 \%$, respectively. Moreover, the prepared CX-g-PHGH paper exhibited an efficient antimicrobial activity against $E$. coli bacterial, by which a lot of 
23

applications based on the new xylan-derived additive agent obtained in this work could be found, especially in field of antimicrobial paper products against $E$. Coli bacteria from contaminated food.

Keywords: antimicrobial additive agent; cationic-xylan; Escherichia coli; mechanical properties; paper products; $\mathrm{PHGH}$; thermal stability

\section{Introduction}

For a long term, the control of harmful bacterial infection has been receiving large amounts of concerns, especially with the increasing quality demand of living environment and working condition in current society ${ }^{[1]}$. Pathogenic Escherichia coli (abbreviated as E. coli) are naturally occurring bacteria found in environment, foods and mostly in intestines of people and animals, which are an important zoonotic bacterial pathogen and could cause a variety of intestinal and extra-intestinal diseases, such as diarrhea, urinary tract infections, septicemia, and neonatal meningitis, and other illnesses $^{[2-6]}$. Contamination of food, specifically meat, with pathogenic E. coli can occur during evisceration and harvest of meat, making it one of the most common causes of food-borne diseases ${ }^{[7,8]}$. Moreover, contact of pathogenic E. coli to human body via different kinds of residing platforms such as food-related paper products, e.g. packaging paper, baking paper, napkin paper, pulp molded tableware, etc. is also a common pathway caused human health problem. Therefore, using antimicrobial products for food-related applications is one of the most efficient and popular ways to 
reduce the infection from the daily eating.

The antimicrobial products based on various raw materials after modification has been extensively studied in the world ${ }^{[9-12]}$. Especially in recent years, antimicrobial products based on biological macromolecules from biomass including lignin, pectin, different kinds of polysaccharides (e.g. cellulose, starch and hemicellulose) have obtained great interests due to many superior properties of these kinds of macromolecules such as innocuousness to human health, biodegradability, good biocompatibility and bioactivity, wide range of resources, easy modification and so on ${ }^{[13-15]}$. For instance, composites of silver-nanoparticles/bacterial-cellulose exhibited significant antibacterial activities against Escherichia coli and other bacteria ${ }^{[16]}$. Antimicrobial nanostructured films based on starch has an antimicrobial activity against Staphylococcus aureus and other bacteria ${ }^{[17]}$.

Food-related cellulosic products, such as food packaging paper, baking paper, napkin paper and so on, are important products in our daily life due to many superior properties of them such as light-weight, easy to modify and use, renewable, biodegradable and so on. However, cellulosic products originally do not have any antimicrobial activity. Bacteria can multiply rapidly in the products if proper conditions were fulfilled. Therefore, there have been many different kinds of natural and synthetic polymers or materials widely studied and used as antibacterial additive agents on these paper products ${ }^{[18-21]}$. Additive of antimicrobial agents especially the agents based on biodegradable biomass on the paper products is a promising way to introduce antimicrobial property to the required materials efficiently and sustainably. 
67

Xylan are the second dominating naturally occurring carbohydrate polymers in plant biomass world after cellulose. They are the most common hemicelluloses in angiosperms, grasses and cereals, where they exist in different composition and structures ${ }^{[22]}$. The content of xylan varies in different species, but it constitutes up to $35 \%$ in sugarcane bagasse and $50 \%$ in straws ${ }^{[23,24]}$. Similar to cellulose, xylan are also poly-hydroxyl polymers with xylose as main sugar unit linked by glycosidic bonds and function as supporting material in the cell wall ${ }^{[25]}$. Xylan are readily available from pulp refining and cereal processing industries, and are at the same time environmentally friendly, renewable, sustainable, biodegradable and biocompatible. Xylan have been found with many potential applications in food, papermaking, textile, plastic industries and biomedical applications ${ }^{[26-28]}$. Moreover, xylan also have great potential to be applied as antimicrobial materials after modification and have received increasing attentions. Recently, the importance of xylan-based macromolecules ${ }^{[29-32]}$ and materials ${ }^{[33-35]}$ have obtained increasing focus, including antimicrobial modification of xylan used in the fields of packaging film, food preservative as well as in biomedical areas ${ }^{[36-39]}$. There have been many ways reported to prepare xylan-based antimicrobial materials. For example, a novel food preservative was prepared by co-heating xylan with chitosan which exhibited excellent antimicrobial activity against Escherichia coli and Staphylococcus aureus ${ }^{[28]}$. Chitosan-xylan/cellulose nanowhiskers (CNW) nanocomposite films with antibacterial and antioxidant properties were successfully prepared where CNW was used as nanofillers ${ }^{[40]}$. Carboxymethylated xylan was blended with Agar (Ag), Ammonium zirconium carbonate (AZC) and linoleic acid (LA) 
to produce edible films with antimicrobial activity ${ }^{[41]}$. Even though, the studies of xylan for high-value added applications are still insufficient. As one of the important subjects, the exploring of possibilities for xylan to be used as antimicrobial additive agent in paper products against $E$. Coli have being focused by researchers.

Same to cellulose, xylan originally do not have antimicrobial activity either, which also requires modification of it to endow the property. Polyhexamethylene guanidine hydrochloride $(\mathrm{PHGH})$ is one kind of water-soluble polycations that has an antimicrobial activity against both Gram positive and Gram-negative bacteria, and low mammalian toxicity ${ }^{[15]}$. The introduction of PHGH could endow macromolecule with efficient antimicrobial activity ${ }^{[42,43]}$ which also offers a potential pathway of endowing xylan with antimicrobial ability against $E$. Coli bacteria.

Therefore, in this work, we prepared a new antimicrobial additive agent based on a xylan-derivative by graft polymerization of PHGH with xylan using ceric ammonium nitrate as initiator. The optimal condition for xylan-derivative preparation was firstly determined by evaluating the whole preparation process. The obtained xylan derivatives were characterized by its structure, thermal stability as well as the rheological behavior determined by Fourier Transform Infrared Spectroscopy (FTIR), Carbon Nuclear Magnetic Resonance Spectroscopy $\left({ }^{13} \mathrm{C}\right.$ NMR), Thermogravimetric Analyser (TGA), Elemental Analyser (EA), and rheology meter. Moreover, mechanical property is also important aspect for the food-related paper products which needs to be concerned. Therefore, the antimicrobial activity against $E$. coli of the xylan-derivative obtained at the optimal condition as well as its ability as strengthening agent to improve mechanical 
111

112

properties of paper sheet were systematically investigated. This work found a new way to modify xylan, and investigated its applications as a new antimicrobial additive agent against E. Coli bacteria and mechanical enhancing agent for paper products. The obtained antimicrobial additive agent product would find great potential applications in many areas, especially for food-related paper products, such as packaging paper, baking paper, napkin paper and so on.

\section{Materials and methods}

\subsection{Materials}

Xylan $\left(M_{\mathrm{w}} 49000 \mathrm{~g} / \mathrm{mol}\right.$, purity $\left.85 \%\right)$ extracted from sugarcane bagasse was obtained from Shanghai Yuanye Bio-Technology Co., Ltd (Shanghai, China). Pretreated waste newspaper pulp (mainly American waste paper) was provided by local paper company (Guangzhou Paper Group Ltd., China). Ceric ammonium nitrate (CAN) (99.0\%, AR) was purchased from Tianjin Damao Chemical Reagent Factory (Tianjin, China). 2, 3-Epoxypropyltrimethylammonium chloride (ETA) (95\%), hexamethylene diamine (98\%, AR), guanidine hydrochloride (99\%, AR), dimethyl sulfoxide ( $\geq 99 \%$, AR) and glycidyl methacrylate (GMA) (97\%, AR) were purchased from Shanghai Macklin Biochemical Co., Ltd (Shanghai, China). Acetone was purchased from Nanjing Chemical Reagent Co., Ltd (Nanjing, China). NaOH (95\%, AR) and Ethanol (99\%, AR) were purchased from Guangzhou Chemical Reagent Factory (Guangzhou, China). Gram negative bacteria (E. coli, ATCC 25922) were purchased from Shanghai 
134

135

Beinuo Bio-Technology Co., Ltd (Shanghai, China). Chemicals used in this study were all analytical reagent grade and used without any purification. Deionized water was used in all experiments. Polyhexamethylene guanidine hydrochloride (PHGH) was prepared by condensation polymerization of hexamethylene diamine and guanidine hydrochloride as described in previous work ${ }^{[19]}$.

\subsection{Preparation of cationic-xylan}

Cationic-xylan $(\mathrm{CX})$ was prepared based on the procedure described in our previous work with minor changes ${ }^{[44]}$. Briefly, a solution of $3 \mathrm{~g}$ xylan in $90 \mathrm{~mL}$ deionized water was prepared in a $250 \mathrm{~mL}$ flask, and followed, $0.736 \mathrm{~g} \mathrm{NaOH}$ (the molar ratio of $\mathrm{NaOH}$ and xylose unit in xylan was 0.8 ) was added for alkalization of xylan for 1 hour. Afterwards, the flask was placed in a microwave reactor (400W) (GAS-800, Beijing Xianghu Science and Technology Development Reagent Co., Ltd., Beijing, China) and $20.907 \mathrm{~g}$ of ETA (the molar ratio of ETA and xylose unit in xylan was 6) was added into the flask when the temperature of microwave reactor was reached to $70^{\circ} \mathrm{C}$. After reaction of $40 \mathrm{~min}$, the precipitate was formed in $100 \%$ ethanol and fractionated by filtration with three times the volume of ethanol. The precipitate was washed by filtration again with $70 \%$ ethanol for a few times until there was no white precipitate formed in the ethanol filtrates by titration with silver nitrate. The washed precipitate was dissolved in deionized water and dialyzed with membrane of molecular weight cut-off of 3500 in DI-water for 5 days until the $\mathrm{pH}$ of the dialyzed liquid reached to neutral. The CX was finally obtained after drying in a vacuum oven 
157

158

159

160

161

162

163

164

165

166

167

168

169

170

171

172

173

under $50^{\circ} \mathrm{C}$ for $24 \mathrm{~h}$. The degree of substitution (DS) of the prepared CX was 0.38 which was determined by elemental analysis method ${ }^{[45]}$.

\subsection{Preparation of cationic-xylan-grafted-PHGH}

Unsaturated double bonds were introduced to PHGH by reacting with GMA where the molar ratio of amino and epoxy groups was kept at $1.0^{[42]}$. The reaction was carried on at room temperature for $6 \mathrm{~h}$. The obtained products were precipitated and washed with acetone to remove unreacted GMA. The washed products were dissolved in appropriate amount of methanol, followed by precipitation and wash with acetone again. The same treatment was performed 3 times, and the final purified GMAmodified PHGH product was obtained after drying in vacuum under $25^{\circ} \mathrm{C}$ for $12 \mathrm{~h}$.

A solution of $0.33 \mathrm{~g} \mathrm{CX}$ in $25 \mathrm{~mL}$ deionized water was prepared in a three-necked round flask $(250 \mathrm{~mL})$ under stirring and purging with nitrogen for $20 \mathrm{~min}$. Then $5 \mathrm{~mL}$ CAN solution was added into the flask with purging of nitrogen continuously for another 10 min. Subsequently, $10 \mathrm{~mL}$ GMA-modified PHGH was added into the CX solution and kept stirring with a slow stream of nitrogen for $4 \mathrm{~h}$. The solution was then dialyzed for 3 days, and the CX-grafted-PHGH (CX-g-PHGH) was finally obtained after drying in a vacuum oven at $50^{\circ} \mathrm{C}$ for $24 \mathrm{~h}$. All conditions of preparing the CX-gPHGH were listed in Table 1. 
Table 1. Influence of the synthesis conditions on CX-g-PHGH

\begin{tabular}{ccccccc}
\hline $\begin{array}{c}\text { Sample } \\
\text { number }\end{array}$ & $\begin{array}{c}\text { Temperature } \\
\left({ }^{\circ} \mathrm{C}\right)\end{array}$ & $\begin{array}{c}\text { PHGH } \\
\text { Concentration } \\
(\mathrm{mol} / \mathrm{L})\end{array}$ & $\begin{array}{c}\text { Initiator } \\
\text { Concentration } \\
(\mathrm{mmol} / \mathrm{L})\end{array}$ & $\begin{array}{c}\text { Time } \\
(\mathrm{h})\end{array}$ & $\begin{array}{c}\text { Graft } \\
\text { ratio } \\
(\%)\end{array}$ & $\begin{array}{c}\text { Graft } \\
\text { Efficiency } \\
(\%)\end{array}$ \\
\hline 1 & 60 & 0.039 & 3 & 4 & 15.54 & 52.54 \\
2 & 60 & 0.039 & 4 & 4 & 18.45 & 58.45 \\
3 & 60 & 0.039 & 5 & 4 & 16.9 & 56.69 \\
4 & 60 & 0.039 & 6 & 4 & 15.93 & 53.35 \\
5 & 60 & 0.020 & 4 & 4 & 7.27 & 56.65 \\
6 & 60 & 0.078 & 4 & 4 & 16.01 & 53.21 \\
7 & 60 & 0.117 & 4 & 4 & 15.26 & 40.73 \\
8 & 60 & 0.156 & 4 & 4 & 15.03 & 36.58 \\
9 & 50 & 0.039 & 4 & 4 & 15.66 & 49.60 \\
10 & 70 & 0.039 & 4 & 4 & 16.82 & 53.28 \\
11 & 80 & 0.039 & 4 & 4 & 16.24 & 51.44 \\
12 & 60 & 0.039 & 4 & 2 & 13.56 & 50.26 \\
13 & 60 & 0.039 & 4 & 3 & 14.18 & 53.47 \\
14 & 60 & 0.039 & 4 & 5 & 17.21 & 57.56 \\
15 & 60 & 0.039 & 4 & 6 & 16.52 & 55.48 \\
\hline
\end{tabular}
on the equations of Eq. (1) and (2), and the mean value of the results obtained from

183 three parallel samples under each condition was reported on Table 1:

184

185
The grafting ratio $=\left(W_{g}-W_{0}\right) / W_{0} \times 100 \%$

The grafting efficiency $=\left(W_{g}-W_{0}\right) / \mathrm{W}_{p} \times 100 \%$

where $W_{0}$ is the weight of CX; $W_{g}$ is the weight of CX-g-PHGH; and $W_{p}$ is the weight of functional PHGH.

\subsection{Characterizations of the prepared products}

Xylan, CX and CX-g-PHGH were dried in an infrared drying oven before characterization for their structures and properties. Fourier Transform Infrared (FT-IR) spectra was obtained with Fourier Transform Spectrophotometer (Nicolet 750, ThermoFisher Scientific, Waltham, FL, USA) appended with Attenuated Total 
195

196

197

Reflectance (ATR) technique. A total of 32 scans were accumulated in the transmission mode, with a resolution of $4 \mathrm{~cm}^{-1}$. The spectrum was obtained from a range of $4000 \mathrm{~cm}^{-}$ ${ }^{1}$ to $400 \mathrm{~cm}^{-1}$.

The solution-state ${ }^{13} \mathrm{C}-\mathrm{NMR}$ spectra was recorded on a Bruker DRX-400 spectrometer (Bruker, Karlsruhe, Germany) at $25^{\circ} \mathrm{C}$ after 15000 scans. The sample (80 $\mathrm{mg}$ ) was dissolved in $1 \mathrm{mLD}_{2} \mathrm{O}$. The running parameters were: $30^{\circ}$ pulse flipping angle, $9.2 \mu \mathrm{s}$ pulse width, $1.36 \mathrm{~s}$ acquisition time with $2 \mathrm{~s}$ relaxation delay.

Element analysis (EA) is for quantitative determination of specific elements of the samples. Specimens weighing approximately 3-5 $\mathrm{mg}$ were heated in a Vario EL Elemental Analyzer (Elementar, Germany) under oxygen atmosphere and elements of $\mathrm{C}, \mathrm{H}$ and $\mathrm{N}$ in xylan, $\mathrm{CX}$ and $\mathrm{CX}-\mathrm{g}-\mathrm{PHGH}$ were determined.

The molecular weights of xylan, CX and CX-g-PHGH were determined by GPC on a PL aquagel-OH 60 column $(300 \mathrm{~mm} \times 7.5 \mathrm{~mm}$, Agilent, USA $)$ and calibrated with PL pullulan polysaccharide standard (average peak molecular weights of 783, 12200, $100000,1600000 \mathrm{~g} / \mathrm{mol})$. A flow rate of $0.5 \mathrm{~mL} / \mathrm{min}$ was maintained with ultrapure water as eluent. Samples were dissolved in ultrapure water to reach a concentration of $0.1 \%$ before characterization.

Dynamic rheological properties of CX and CX-g-PHGH were determined by a sandwich rheometer (AR2000, TA Instruments, New Castle, DE, USA). All samples were dissolved in water with a magnetic stirrer for $30 \mathrm{~min}$ to form stable solutions. The solutions were then dropped on Brookfield D VIII instrument panel. A software (Rheo 2000) provided by the manufacturer with the instrument was used to setup the 
217

218

parameter, perform rheometer control and collect data. The data of shear rate, frequency, viscosities, storage modulus (G') and the loss modulus (G") of all samples were collected.

Thermal stability analysis was used to determine thermodynamic properties of xylan, CX and CX-g-PHGH. The analysis was carried out using thermogravimetric analysis (TGA) and differential thermal analysis (DTA) on a simultaneous thermalgravimetric analyzer (TGA Q500, TA Instruments, New Castle, USA). About 5 mg samples were heated to $700^{\circ} \mathrm{C}$ from room temperature with the heating rate of $10^{\circ} \mathrm{C} / \mathrm{min}$ in a nitrogen atmosphere.

\subsection{Preparation and mechanical properties tests of $C X$-g-PHGH paper sheets}

Five sheets with grammage (weight per unit area) of $\sim 55 \mathrm{~g} / \mathrm{m}^{2}$ were prepared based on norms GB2828-81 (Chinese Technical Association of Pulp and Paper). The mass ratio of CX-g-PHGH and the waste newspaper pulp was 3 15:1000. Briefly, CXg-PHGH and the waste newspaper pulp were homogenized under stirring for $10 \mathrm{~min}$ before forming paper sheets. The sheets were formed via a fast papermaking machine (MESSMER 255, USA) and dried. The obtained sheets were cut to a certain shape and placed in humidity room at $25^{\circ} \mathrm{C}$ for $24 \mathrm{~h}$ before mechanical tests. As comparison, CX paper sheets were also produced via the same preparation process. A Chinese Standard (Chinese Technical Association of Pulp and Paper) was applied for mechanical tests of the sheets. The tests were carried out 10 times for each sample, and the mean value of the mechanical tests was reported in this work. 
2.6. Antimicrobial tests of the xylan derivatives

242

The antimicrobial activity was tested against Gram negative bacteria $(E$. coli,

ATCC 25922). The E. coli bacteria were cultured and grown in Luria Bertani (LB)

245 liquid medium (10 g/L peptone, $5 \mathrm{~g} / \mathrm{L}$ yeast extract, $10 \mathrm{~g} / \mathrm{L} \mathrm{NaCl}$, and at $\mathrm{pH} 7.0)$ for 12

246

247

h at $37^{\circ} \mathrm{C}$. The bacteria were further diluted with $\mathrm{NaCl}$ solution $(0.85 \%$, w/v) to get concentration of about $10^{5} \mathrm{CFU} / \mathrm{mL}$. The diluted suspensions $(0.1 \mathrm{~mL})$ of $E$. coli were then distributed homogeneously onto the LB agar medium $(10 \mathrm{~g} / \mathrm{L}$ peptone, $5 \mathrm{~g} / \mathrm{L}$ yeast extract, $10 \mathrm{~g} / \mathrm{L} \mathrm{NaCl}$, and $15 \mathrm{~g} /$ lagar). The obtained paper sheets with xylan-derivatives in diameter of 6-mm was prepared and placed in the plate culture medium that was coated with the bacteria suspension. The antimicrobial ability of the xylan-derivatives was evaluated by measuring the diameter of the inhibition zones to $E$. coli on the paper sheets.

\section{Results and Discussion}

\subsection{Synthesis determination of $C X-g-P H G H$}

The $\mathrm{Ce}^{4+}$ in ceric ammonium nitrate could attack and convert the hydroxyl groups of CX to free radicals, which could have activity to further react with modified PHGH and form CX-g-PHGH ${ }^{[46]}$. The synthesis procedure was proposed in Scheme I. 
<smiles>COC1OCC(C)C(OCC(O)N(C)C)C1O</smiles><smiles>COC1OCC(C)C(OCC(O)N(C)C)C1[O-]</smiles><smiles>C=C(C)C(=O)OCC(O)CNP</smiles>

Scheme I. Copolymerization of PHGH onto CX.

There are many factors in the reaction such as the concentration of initiator, amounts

Figure 1a and detailed values were shown in Table 1.
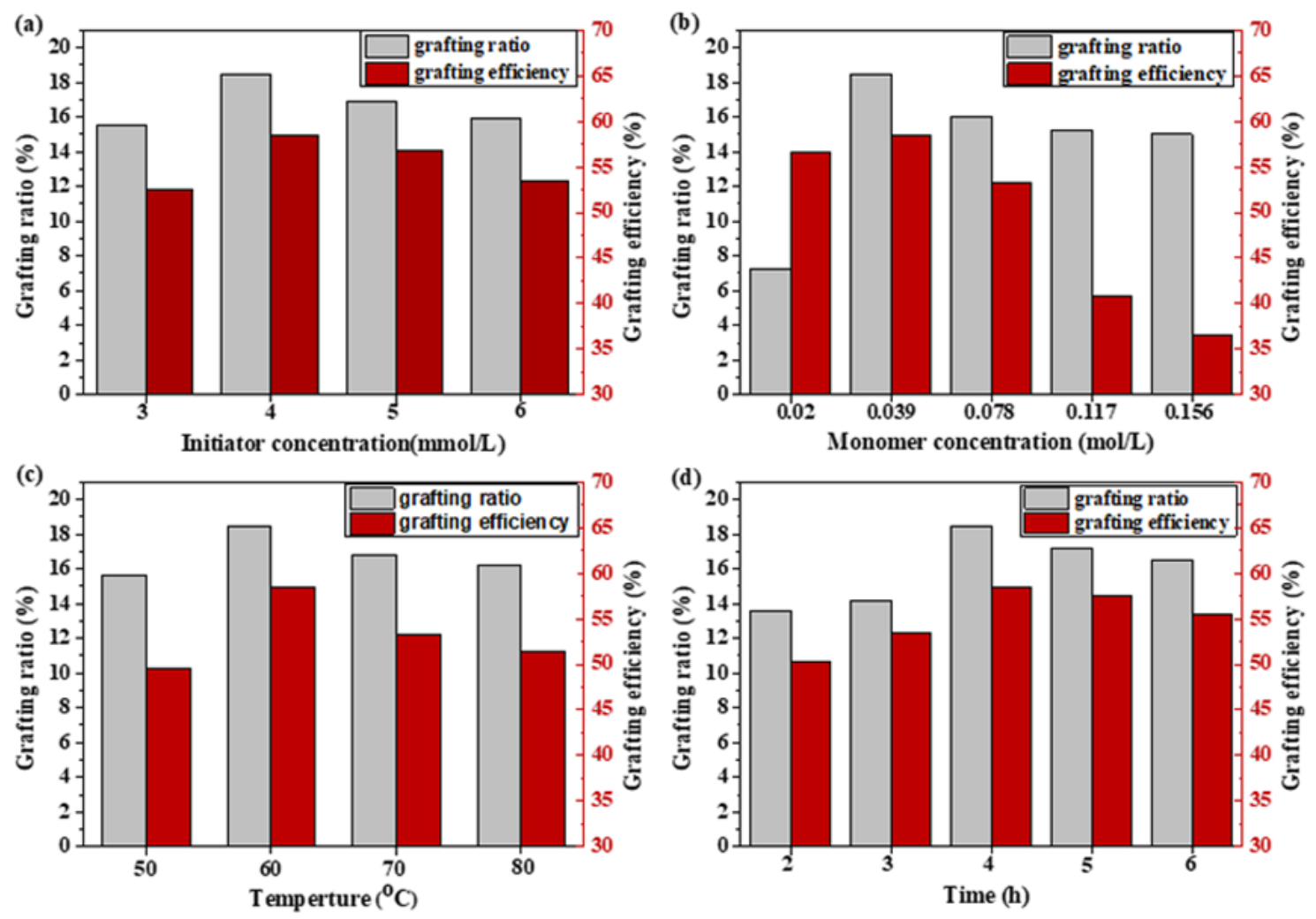
270

271

273

274

Figure 1. Influences of conditions on grafting ratio and efficiency of CX-g-PHGH, a) initiator concentration; b) PHGH concentration; c) temperature; and d) time.

As it is shown that grafting ratio and efficiency increased drastically with the increase of initiator concentration until $4 \mathrm{mmol} / \mathrm{L}$, valued up to about $18.45 \%$ and $58.45 \%$ for grafting ratio and efficiency, respectively. However, when higher initiator concentration than $4 \mathrm{mmol} / \mathrm{L}$ was applied, both the grafting ratio and efficiency decreased. This phenomenon was in accordance to previous finding by Qian, which was probably because the excess initiator started to participate in the termination step of the growing chains and subsequentially initiated the homopolymerization of PHGH $[18]$.

The influence of PHGH concentration on the grafting ratio and grafting efficiency was shown in Figure 1b. It is very clear to see that the grafting ratio and efficiency increased sharply up to $18.45 \%$ and $58.45 \%$, respectively, when the PHGH concentration was lower than $0.04 \mathrm{~mol} / \mathrm{L}$. However afterwards, the grafting ratio and efficiency decreased rapidly which was probably caused by the self-polymerization resulted from high PHGH concentration ${ }^{[15]}$.

The increase of reaction temperature may lead to multiple effects, including increasing the diffusion of $\mathrm{CX}$ and $\mathrm{PHGH}$; facilitating redox initiator system and enhancing the chain propagation, but likely increasing the rate of termination and homopolymerization in bulk phase ${ }^{[47,48]}$. Therefore, when the temperature was lower than $60^{\circ} \mathrm{C}$, there was a sharp increase for both grafting ratio and efficiency reached to maximum value of $18.45 \%$ and $58.45 \%$, respectively (Figure 1c). However, the grafting 
292

293

ratio and efficiency decreased when the reaction temperature was higher than $60^{\circ} \mathrm{C}$ which was due to the domination of homopolymerization caused by high temperature. Furthermore, the reaction time also had important impact on the grafting ratio and efficiency of CX-g-PHGH as it is shown in Figure 1d. When the reaction time increased, the grafting ratio and the efficiency firstly increased and then decreased. Reaction time of $4 \mathrm{~h}$ was the optimal time for the grafting ratio and efficiency with maximum value of $18.45 \%$ and $58.45 \%$, respectively. This can be explained by the decrease of PHGH concentration and free radicals in the system as the increase of reaction time, which resulted in the leveling off of the grafting ratio and efficiency ${ }^{[49]}$.

It can be concluded from above that the optimal condition for preparing CX-gPHGH were: initiator concentration $4 \mathrm{mmol} / \mathrm{L}$; $\mathrm{PHGH}$ concentration $0.039 \mathrm{~mol} / \mathrm{L}$; reaction temperature $60^{\circ} \mathrm{C}$ and reaction time 4 hours. Therefore, in the following studies, the CX-g-PHGH (sample number 2, as CX-g-PHGH-1) obtained at the optimal condition with maximal grafting ratio of $18.45 \%$ was used for characterizations and tests.

\subsection{FTIR Spectra}

In order to confirm the successful grafting of PHGH on CX, several analyses, including spectra of FTIR and ${ }^{13} C-N M R$, element analysis and average molecular weight, were performed.

The FTIR spectra of xylan, CX and CX-g-PHGH-1 are illustrated in Figure 2. 
315

the spectrum represent the transmittance peaks of molecular bonds for xylan. The transmittance band at $2918 \mathrm{~cm}^{-1}$ is assigned to the $\mathrm{C}-\mathrm{H}$ stretching vibration of alkane in xylan. The transmittance peak of $1044 \mathrm{~cm}^{-1}$ is ascribed to the C-O-C stretching of the ether groups ${ }^{[44]}$.

(a)

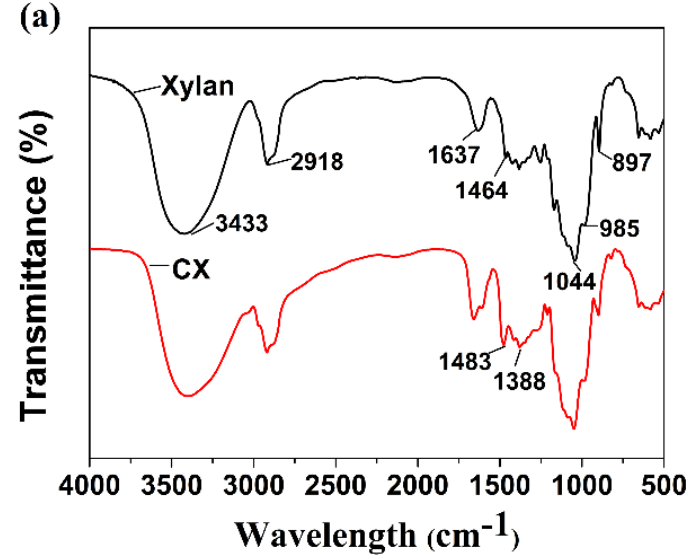

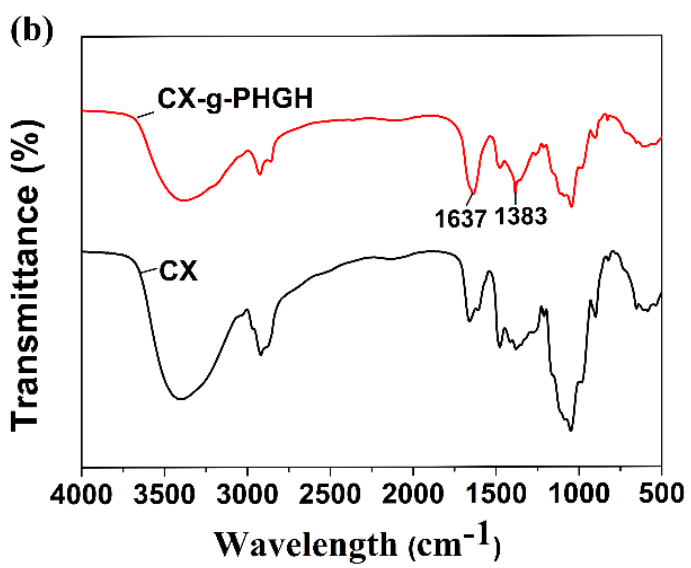

Figure 2. FTIR spectra of (a) xylan and CX; (b) CX and CX-g-PHGH-1.

As we can see from Figure 2a, in the FTIR spectra of CX, an enhancement in the transmittance intensity of ether bond at $1044 \mathrm{~cm}^{-1}$ was found compared with the spectrum of xylan, indicating that more ether bonds were introduced to xylan. The transmittance intensity around $1388 \mathrm{~cm}^{-1}$ increased due to stretching vibration of C-N. The stretching vibration of $-\mathrm{CH}_{2}$ and $-\mathrm{CH}_{3}$ on the quaternary ammonium group enhanced the intensity of the transmittance at $1483 \mathrm{~cm}^{-1}$. All these changes in transmittance peaks indicated that cationic groups were introduced to xylan successfully ${ }^{[50]}$.

In Figure 2b, compared with the spectrum of $C X$, enhancement in the intensities of transmittance at $1383 \mathrm{~cm}^{-1}$ and $1637 \mathrm{~cm}^{-1}$ was discovered in the FTIR spectra of CXg-PHGH, corresponding for the stretching vibration transmittance peaks of $\mathrm{C}-\mathrm{N}, \mathrm{C}=\mathrm{N}$, respectively which confirmed the successful introduction of $\mathrm{PHGH}$ to $\mathrm{CX}^{[42]}$. 

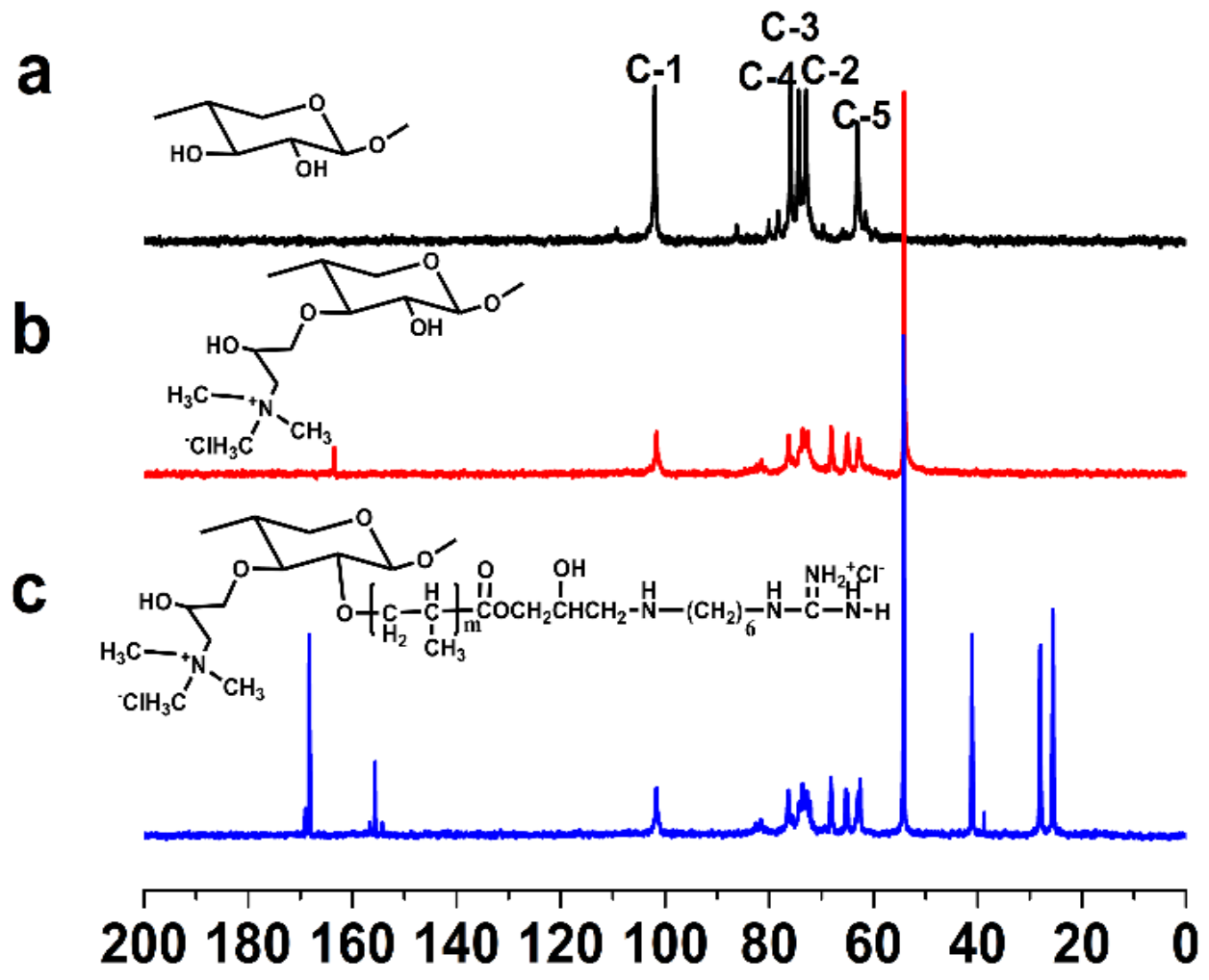

Figure 3. ${ }^{13} \mathrm{C}-\mathrm{NMR}$ spectra of a) xylan, b) $\mathrm{CX}$ and c) CX-g-PHGH-1. 
346

347

348

349

350

351

352

353

354

355

356

357

358

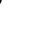

moiety and the signal at $68.1 \mathrm{ppm}$ is attributed to the carbon $\mathrm{C}\left(\mathrm{CH}_{2}\right)^{[51]}$. This proved that the cationic groups were introduced successfully onto xylan. In ${ }^{13} \mathrm{C}-\mathrm{NMR}$ spectrum of CX-g-PHGH-1 (Fig. 3c), some new peaks appeared in comparison with CX. The peaks at $28.0 \mathrm{ppm}, 41.1 \mathrm{ppm}$ and $168 \mathrm{ppm}$ are attributed to the signal peaks of $-\mathrm{CH}_{2}$, C-N and ester group carbon ${ }^{[52]}$, respectively, which indicated that PHGH was grafted onto CX successfully.

\subsection{Element analysis}

The element analysis of xylan, CX and CX-g-PHGH-1 are showed in Table 2. The contents $\mathrm{C}, \mathrm{H}$ and $\mathrm{N}$ in xylan were $40.36 \%, 6.91 \%$ and $0.00 \%$, respectively, while they became $40.36 \%, 7.43 \%$ and $2.16 \%$ after the cationic modification of xylan, indicating that cationic groups were grafted on xylan.

Table 2. The element analysis of xylan, CX and CX-g-PHGH-1.

\begin{tabular}{cccc}
\hline Sample & $\mathrm{C}(\%)$ & $\mathrm{H}(\%)$ & $\mathrm{N}(\%)$ \\
\hline Xylan & 40.36 & 6.91 & 0 \\
CX & 40.36 & 7.43 & 2.16 \\
CX-g-PHGH & 40.88 & 7.79 & 9.46 \\
\hline
\end{tabular}

Furthermore, the contents of C, $\mathrm{H}$ and $\mathrm{N}$ in CX-g-PHGH-1 were $40.88 \%, 7.79 \%$ and $9.46 \%$, respectively. The increase in the $\mathrm{N}$ content confirmed the presence of nitrogenous compounds (PHGH) in CX-g-PHGH-1.

\subsection{Average Molecular Weight}


367

successful conversion of xylan to its derivatives. The molecular weight and molecular weight distribution of xylan, CX and CX-g-PHGH-1 are shown in Table 3.

Table 3. The molecular weight and molecular weight distribution of xylan, CX and CX-g-PHGH-1.

\begin{tabular}{ccc}
\hline Sample & $M_{\mathrm{w}}(\mathrm{g} / \mathrm{mol})$ & $M_{\mathrm{w}} / M_{\mathrm{m}}$ \\
\hline xylan & 49000 & 4.59 \\
CX & 37500 & 1.99 \\
CX-g-PHGH-1 & 1477129 & 1.15 \\
\hline
\end{tabular}

As it is shown obviously, the molecular weight of CX was lower than that of xylan, which was probably caused by the degradation of xylan during chemical reaction under alkaline conditions. As expected, the molecular weight of CX-g-PHGH-1 was higher, being about 30-40 folds than that of xylan and CX, which indicated the successful copolymerization of xylan and CX with PHGH. In addition, CX and CX-gPHGH-1 had a relatively low index of polydispersity (1.14-1.99) than xylan (4.59), which indicated that the molecular chain length distribution of CX and CX-g-PHGH-1 was more uniform than the one of xylan.

\subsection{Rheological properties}

Properties of xylan, CX, CX-g-PHGH-1 as well as their influences to paper products were tested in order to fulfill the requirements used as additive agent for antimicrobial paper products.

The tests of rheological behavior for xylan, CX and CX-g-PHGH-1 would provide better understandings of the physico-chemical properties of the polymers and consequently discover their potential applications, e.g. as coating additive agent to 
388 389

390

paper products. The rheological behavior of xylan, $\mathrm{CX}$ and $\mathrm{CX}-\mathrm{g}-\mathrm{PHGH}-1$ are shown in Figures 4 and 5.
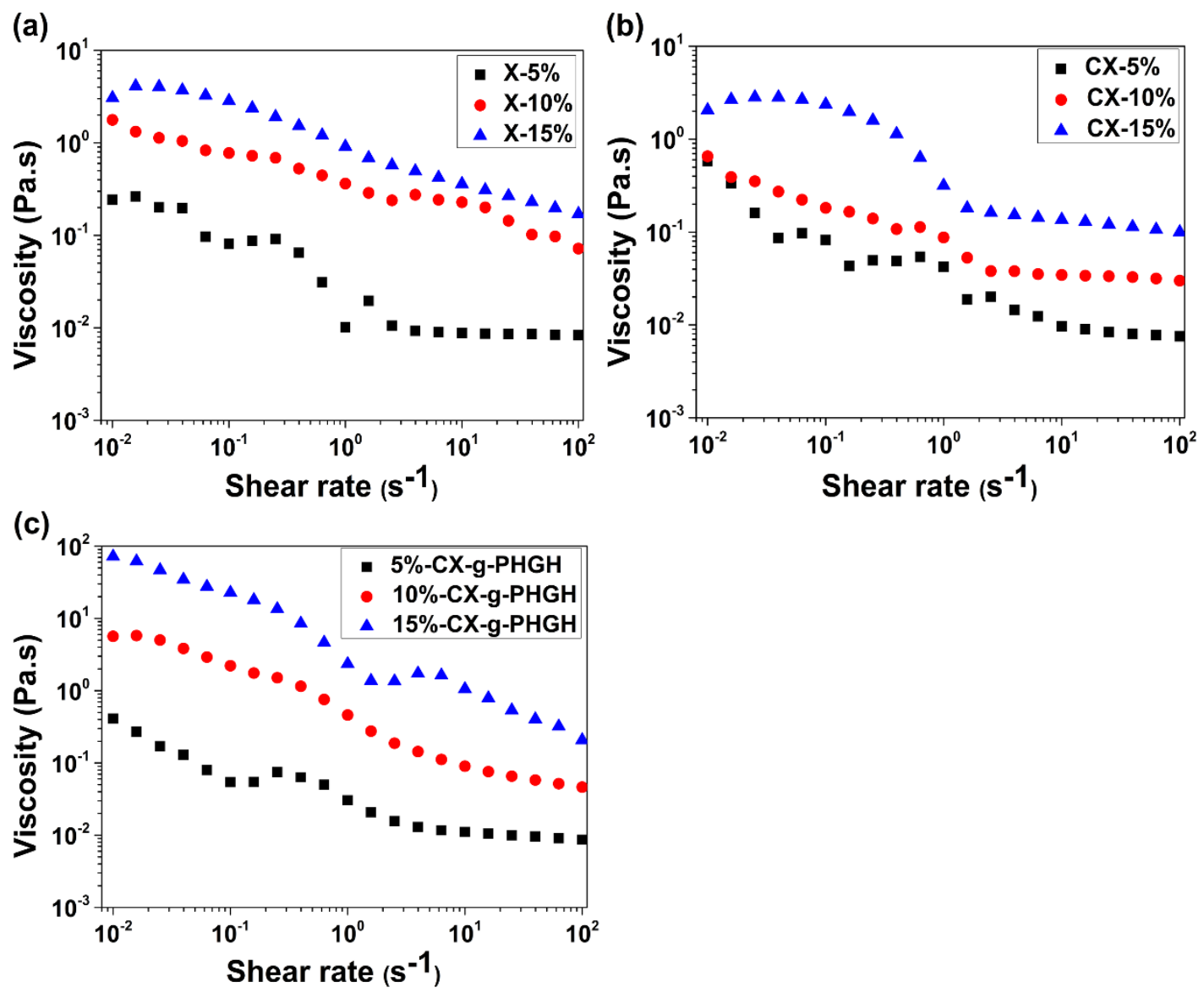

Figure 4. Shear rate dependence of viscosity for (a) xylan, (b) CX and (c) CX-g-PHGH1 at different concentrations.

As it can be seen from Figure 4, viscosities of xylan, CX and CX-g-PHGH-1 all decreased with the increase of shear rate, exhibited pseudoplastic or shear-thinning behavior of these solutions in the range of shear rates tested, which was due to the damage of network structure of xylan and its derivatives ${ }^{[45]}$. Accordingly, with a certain additive speed, xylan, CX and CX-g-PHGH-1 would be easily applied as coating agent on the surface of materials such as paper materials. Furthermore, the viscosity of CXg-PHGH-1 solution was higher than that of xylan and CX solutions in the whole shear 
400

401

402

403

404

405

406

407

408

409

410

411

412

413

414

415

416

rates range when the concentrations were $5 \%$ and $10 \%$ as well as for higher shear rate than $10 \mathrm{~s}^{-1}$ when the concentration was $15 \%$. This can be suggested that CX-g-PHGH1 solution had stronger intermolecular interactions than other two solutions ${ }^{[53]}$.

(a)

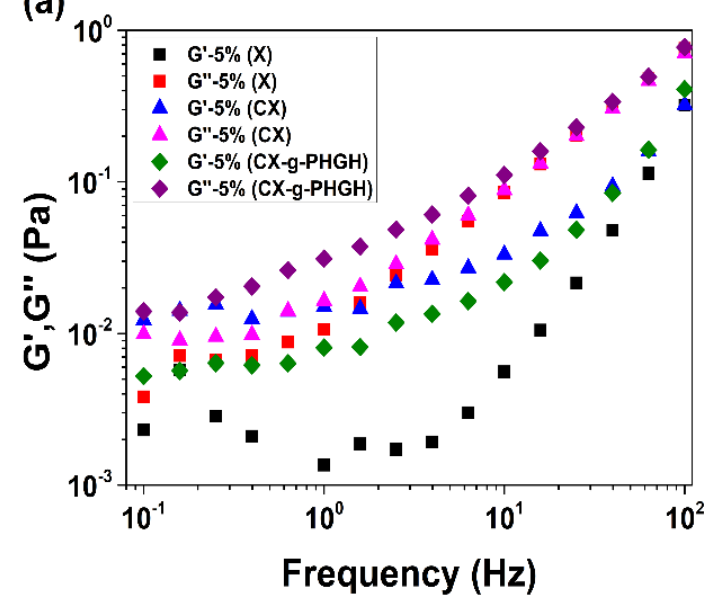

(b)

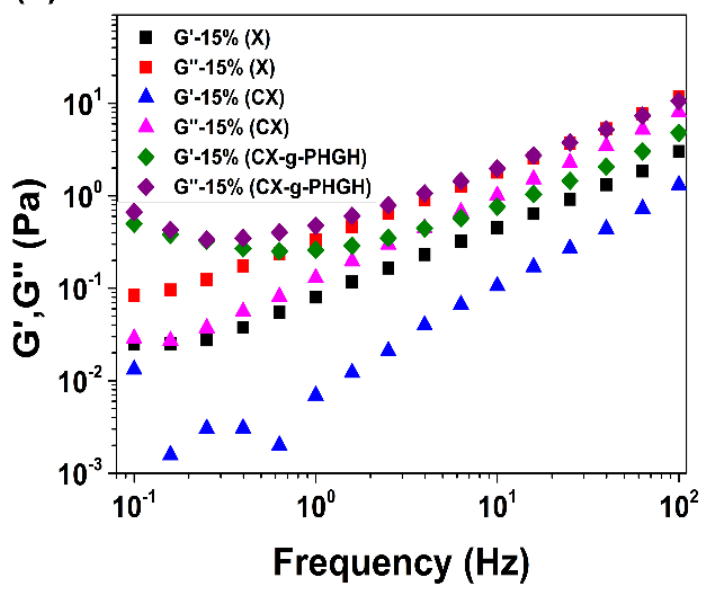

Figure 5. Frequency dependent modulus of the solutions of xylan (X), CX and CX-gPHGH-1 (a) at 5\% concentration; (b) at $15 \%$ concentration.

In Figure 5, the rheological properties (storage modulus G' and loss modulus G”) of xylan, CX and CX-g-PHGH-1 are illustrated. When the concentration was 5\%, the storage modulus in the whole frequency region of xylan and CX-g-PHGH-1 solutions was lower than the loss modulus, exhibiting a viscous behavior. For CX solution with concentration of $5 \%$ in the range of $10^{-1}$ to $10^{0} \mathrm{~Hz}$, the storage modulus was higher than the loss modulus, showing stronger elastic properties due to stronger molecule entanglement of CX than xylan and CX-g-PHGH-1 ${ }^{[45]}$. When the concentration of solution was $15 \%$, all storage modulus of xylan, CX and CX-g-PHGH-1 in the whole frequency region were lower than that of loss modulus, showing a viscous behavior. In addition, when the concentration of solution was $15 \%$, the storage and loss modulus of CX-g-PHGH-1 was higher than the modulus of xylan and CX, indicating a greater 
417

418

419

420

421

422

423

424 changed the dynamic shear rheological properties of $\mathrm{CX}^{[54]}$. The studies above not only

425 further confirmed the successful grafting of PHGH to xylan, but also indicated that the

viscosity behavior for CX-g-PHGH-1 than xylan and CX.

These results can be explained by the influence of molecular weight and functional groups of macromolecule chains ${ }^{[45]}$. Higher molecular weight and guanidine group caused more chain segments needed for the movement of the viscous flow, thus increased the frictional resistance and showed a greater viscosity behavior. On the other hand, lower molecular weight as well as cationic groups on the polymer chain may reduce or prevent the associative interactions among themselves in solutions, and thus xylan-derivative obtained in this work is a promising candidate as coating additive agent which can be applied for paper products.

\subsection{Thermal stability analysis}

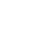

The thermal stability analysis of the samples could facilitate the application of xylan-derivative in more scopes. The typical TGA/DTA curves of xylan, CX and CXg-PHGH-1 are displayed in Figure 6. There was a weight loss starting from $200^{\circ} \mathrm{C}$ which corresponded to the evaporation of water from the samples. 


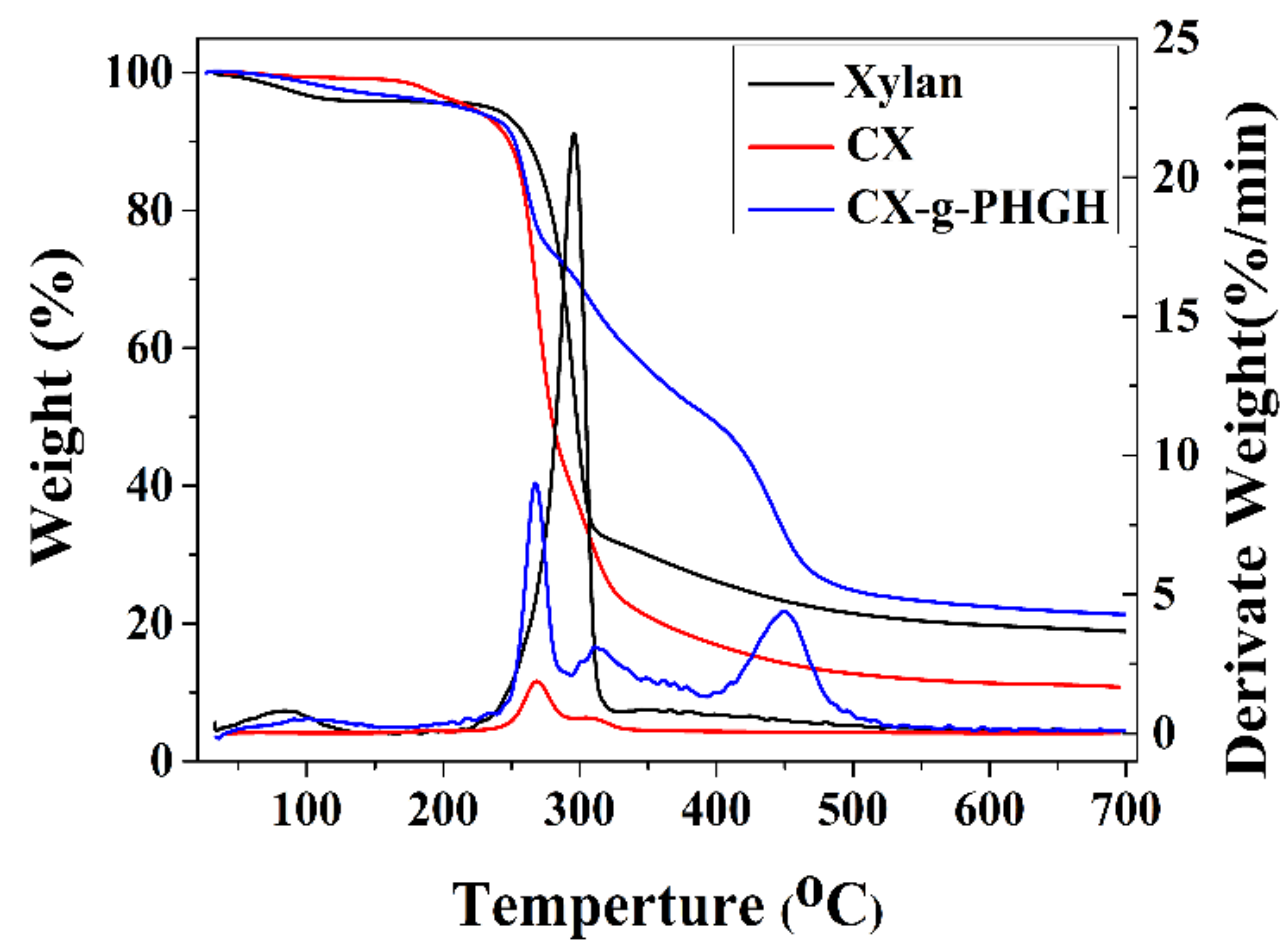

435

436

Figure 6. The TGA/DTA curves of xylan, CX and CX-g-PHGH-1.

It can be seen that the substantial weight loss of xylan occurred at $250-320^{\circ} \mathrm{C}$,

which could be attributed to backbone scission and following fragmentation of xylan

[52]. The CX exhibited a similar thermal stability pattern to that of xylan, but its

decomposition temperature was lower, indicating that $\mathrm{CX}$ was more unstable than xylan.

This is the fact that the hydrogen bonds and molecular structure were destroyed to a

certain extent after cationization, and the grafted cationic groups were not stable ${ }^{[44.49]}$.

Similar to the TGA curves of xylan and CX, there was also a slight loss below $200^{\circ} \mathrm{C}$

for the CX-g-PHGH-1 which was attributed to surface water evaporation. A substantial weight loss of CX-g-PHGH-1 was found in the range of $230-320^{\circ} \mathrm{C}$ which was due to the thermal decomposition of xylan. A substantial weight loss of CX-g-PHGH-1 was also occurred in the range of $320-470^{\circ} \mathrm{C}$ which was attributed to the thermal decomposition of $\mathrm{PHGH}$. Above $470^{\circ} \mathrm{C}$, the polymer began to carbonize and the weight 
449 of matter tends to be constant gradually ${ }^{[55]}$. As a whole, the derivatization of xylan with 450 PHGH endowed the new xylan-derivative with sufficient thermal stability which is 451 beneficial for it to be used under high temperature, such as producing paper and 452 container products, baking foods, packaging hot foods and so on.

453

3.8. Influence of xylan-derivatives addition on mechanical properties of paper sheets

455

456

457

458

459

460

461

462

463

464

465

466

467

468

The mechanical properties of the paper products are important in the aspect of applications in packaging, baking, cleaning and so on. The influences of xylan, CX and CX-g-PHGH-1 addition on mechanical properties of the paper sheets are shown in Table 4, 5 and 6, respectively.

Table 4. Mechanical properties of paper sheets after addition of xylan.

\begin{tabular}{ccccc}
\hline $\begin{array}{c}\text { Amount of } \\
\text { xylan }(\mathrm{wt} \%)\end{array}$ & $\begin{array}{c}\text { Tear index } \\
\left(\mathrm{mN} \cdot \mathrm{m}^{2} / \mathrm{g}\right)\end{array}$ & $\begin{array}{c}\text { Burst index } \\
\left(\mathrm{kPa} \cdot \mathrm{m}^{2} / \mathrm{g}\right)\end{array}$ & $\begin{array}{c}\text { Tensile index } \\
(\mathrm{Nm} / \mathrm{g})\end{array}$ & $\begin{array}{c}\text { Folding } \\
\text { endurance } \\
(\text { times })\end{array}$ \\
\hline 0 & 6.36 & 2.02 & 35.67 & 9 \\
0.3 & 6.39 & 2.10 & 36.83 & 9 \\
0.5 & 6.42 & 2.18 & 37.26 & 10 \\
1.0 & 6.45 & 2.33 & 39.17 & 12 \\
1.5 & 6.40 & 2.24 & 38.23 & 11 \\
\hline
\end{tabular}

Table Footnote: Quantitative of each sheet was approximately $55 \mathrm{~g} / \mathrm{m}^{2}$, the stirring time of pulp after addition of xylan was $10 \mathrm{~min}$.

It is obviously to see from Table 4, that addition of xylan had only a little effect on all mechanical properties of the paper sheets listed in the table. When the amount of xylan was $1.0 \mathrm{wt} \%$ in paper sheet, compared to the mechanical properties of reference paper without xylan addition, the index of tensile, tear and burst were increased by $9.81 \%, 1.42 \%$ and $15.35 \%$, respectively. Meanwhile, there was also a minor increase for the folding endurance after addition of xylan. 
469 Table 5. Mechanical properties of paper samples after addition of CX.

\begin{tabular}{ccccc}
\hline $\begin{array}{c}\text { Amount of } \\
\begin{array}{c}\text { Cationic-xylan } \\
(\mathrm{wt} \%)\end{array}\end{array}$ & $\begin{array}{c}\text { Tear index } \\
\left(\mathrm{mN} \cdot \mathrm{m}^{2} / \mathrm{g}\right)\end{array}$ & $\begin{array}{c}\text { Burst index } \\
\left(\mathrm{kPa} \cdot \mathrm{m}^{2} / \mathrm{g}\right)\end{array}$ & $\begin{array}{c}\text { Tensile index } \\
(\mathrm{Nm} / \mathrm{g})\end{array}$ & $\begin{array}{c}\text { Folding } \\
\text { endurance } \\
(\text { times })\end{array}$ \\
\hline 0 & 6.36 & 2.02 & 35.67 & 9 \\
$0.3 \%$ & 6.44 & 2.26 & 37.45 & 9 \\
$0.5 \%$ & 6.65 & 2.38 & 38.89 & 10 \\
$1.0 \%$ & 7.12 & 2.51 & 40.48 & 14 \\
$1.5 \%$ & 6.89 & 2.31 & 39.06 & 12 \\
\hline
\end{tabular}

470

Table Footnote: Quantitative of each paper was approximately $55 \mathrm{~g} / \mathrm{m}^{2}$, the stirring time of pulp after addition of CX was10 min.

The addition of CX also improved the mechanical properties of the paper sheets but to a larger extent than addition of xylan which can be seen from Table 5. This was probably because the cationic groups in $\mathrm{CX}$ could absorb the anionic groups $(-\mathrm{OH})$ on the fibers, which could improve the combined forces among fibers, thereby increased the mechanical properties of sheets ${ }^{[56]}$. Compared to the reference paper sheet, when the amount of CX was $1.0 \mathrm{wt} \%$, the index of tensile, tear and burst were increased by $13.48 \%, 11.95 \%$ and $24.26 \%$, respectively, and folding endurance was increased by $55.55 \%$. The mechanical properties of paper sheets became lower when the addition of CX was over $1.0 \mathrm{wt} \%$. This was probably caused by excessive addition of cationic group in CX which prevented hydrogen bonding between cellulose fibers, correspondingly resulted in the decrease of the paper strength ${ }^{[43]}$.

When CX-g-PHGH was added into the paper sheets, there were obvious improvements to the mechanical properties of the paper sheets which was affected not only by the amount of addition but also the grafting ratios of PHGH (Table 6).

Table 6. Mechanical properties of paper samples after addition of CX-g-PHGH.

\begin{tabular}{cccccc}
\hline Grafting & Amount of & Tear index & $\begin{array}{c}\text { Burst index } \\
\left(\mathrm{kPa} \cdot \mathrm{m}^{2} / \mathrm{g}\right)\end{array}$ & $\begin{array}{c}\text { Tensile } \\
\text { index }\end{array}$ & $\begin{array}{c}\text { Folding } \\
\text { endurance }\end{array}$ \\
\hline
\end{tabular}




\begin{tabular}{cccccc}
\hline & $\begin{array}{c}\text { PHGH } \\
(\text { wt \%) }\end{array}$ & & & $(\mathrm{Nm} / \mathrm{g})$ & (time) \\
\hline 0 & 0 & 6.36 & 2.02 & 35.67 & 9 \\
14.27 & 1 & 7.35 & 2.48 & 40.96 & 12 \\
15.54 & 1 & 7.84 & 2.59 & 41.97 & 15 \\
16.90 & 1 & 7.73 & 2.60 & 42.00 & 15 \\
18.45 & 1 & 7.97 & 2.63 & 42.83 & 16 \\
18.45 & 0.3 & 6.71 & 2.22 & 40.35 & 13 \\
18.45 & 0.5 & 7.35 & 2.57 & 40.97 & 14 \\
18.45 & 1.5 & 7.26 & 2.55 & 41.23 & 12 \\
\hline
\end{tabular}

487

488

489

490

491

492

493

494

495

496

497

498

499

500

501

502

503

504

Table Footnote: Quantitative of each paper was approximately $55 \mathrm{~g} / \mathrm{m}^{2}$, the stirring time of pulp after addition of CX-g-PHGH-1 was 10 min.

As it is shown clearly in Table 6, CX-g-PHGH with higher grafting ratio led to higher mechanical properties of paper. When CX-g-PHGH-1 (the highest grafting ratio $18.45 \%$ obtained at optimal condition) was added, compared with the reference paper sheet, the index of tensile, tear, burst and folding endurance increased by $20.07 \%$, $25.31 \%, 30.20 \%$ and $77.78 \%$, respectively. It is also well-known that paper strength is dependent on the fiber strength as well as primarily the hydrogen bonding force among fibers. PHGH contains guanidine group with positive charge which endows PHGH ability to adsorb onto fibers with anionic group ${ }^{[15]}$. Therefore, the improvements of mechanical properties were explained probably by such: when CX-g-PHGH was added into the paper pulp, CX-g-PHGH filled or adhered to the space between fibers which resulted in the increase of the bonding point and bonding area between fibers, correspondingly improved the mechanical properties of sheets ${ }^{[57]}$. Moreover, the guanidine group and cationic groups of CX-g-PHGH adsorbed negative charge of fibers, which thereby resulted in the increase of fiber retention, improvement of the bond between fibers and correspondingly improved the mechanical properties of paper sheets ${ }^{[56]}$. The mechanical properties of the paper samples were improved as the increase of 
505

506

507

508

509

510

511

512

513

514

515

516

517

CX-g-PHGH-1 addition. Furthermore, the amount of added CX-g-PHGH-1 also affected the mechanical properties of the paper samples. However, the mechanical properties of paper began to decrease when the amount of added CX-g-PHGH-1 was over $1.0 \mathrm{wt} \%$. This can be explained that the excessive $\mathrm{NH}_{2}$ brought from CX-g-PHGH1 prevented the hydrogen bonding between cellulose fibers, which resulted in the decrease of the paper strength ${ }^{[43]}$.

\subsection{Antimicrobial test of the paper sheets with addition of $C X$ and $C X-g-P H G H$}

In this work, the antimicrobial activity of the paper sheets with addition of the xylan-derivatives was evaluated against $E$. coli bacteria by inhibition zone method and shown in Figure 7. As comparison, the antimicrobial activity tests for CX-g-PHGH (sample number 1 in Table 1, as CX-g-PHGH-2) obtained with lower initiator concentration and a relative lower grafting ratio of $15.54 \%$ was also studied.

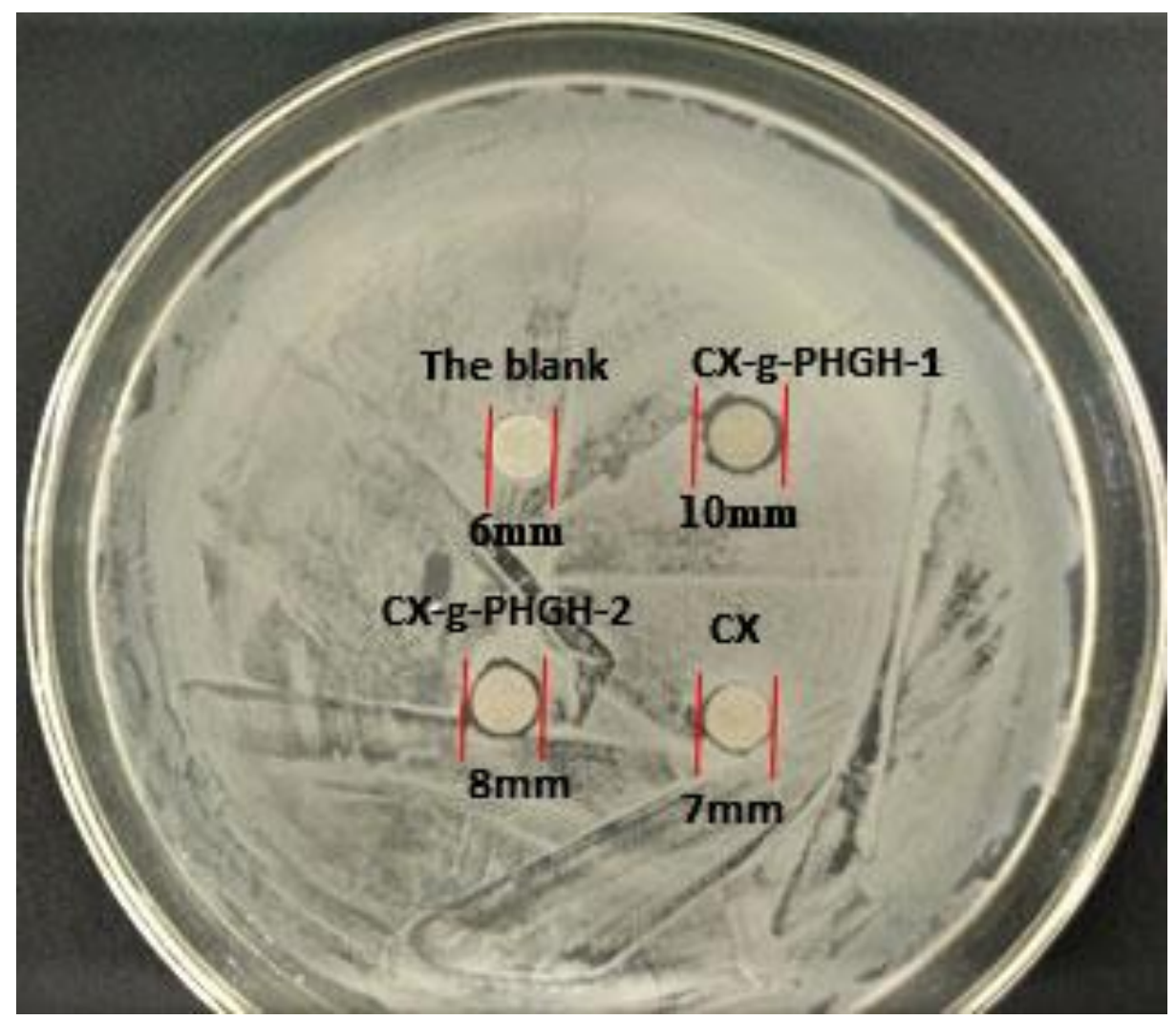


520 Figure 7. The antimicrobial activity of the paper samples with the addition of CX, CX-

521 g-PHGH-1, CX-g-PHGH-2 against E. coli bacteria.

Inhibition zone method is the most used method for antimicrobial activity tests

523 of materials. By using this method, the growth of bacterial was inhibited in the

524 formation of transparent circles by diffusion of antimicrobial agents in agar plates. The

525 antimicrobial ability of the agents was evaluated by the size of the inhibition circle.

526 Guanidino groups of guanidine polymers adsorbed the anions on the cell surface of $E$.

527 Coli bacterial by electrostatic action which following destroyed normal metabolism and

528 surface structure of the living bacterial cells, sequentially inhibited the growth of

529 bacteria effectively ${ }^{[18]}$.

530 It can be seen that addition of $\mathrm{CX}$ and $\mathrm{CX}$-g-PHGH endowed paper sheet

531 antimicrobial property. CX paper sample showed weaker antimicrobial ability than CX-

532 g-PHGH samples with smaller inhibition zone diameter of $7 \mathrm{~mm}$. The diameter of

533 inhibition zone for the paper of CX-g-PHGH-1 (grafting ratio 18.45\%) and CX-g-

534 PHGH-2 (grafting ratio 15.54\%) against E. coli enlarged from $6 \mathrm{~mm}$ to 10 and $8 \mathrm{~mm}$,

535 respectively, which suggested that the CX-g-PHGH paper had excellent antimicrobial

536 activity against E. coli and the antimicrobial activity was improved by the increase of

537 PHGH contents grafted on the CX.

\section{Conclusions}

540

In present study, a novel xylan-based antimicrobial additive agent was 
542

543

544

successfully prepared and applied in cellulosic paper sheets with improved mechanical properties and antimicrobial activity against E. Coli bacteria. The derived xylan, cationic xylan-grafted-PHGH (CX-g-PHGH), was successfully synthesized by graft copolymerization of cationic xylan (CX) with guanidine polymer (PHGH) using ceric ammonium nitrate (CAN) as initiator. The optimal reaction parameters for obtain efficiently the CX-g-PHGH were $4 \mathrm{~h}$ at $60^{\circ} \mathrm{C}$ with $\mathrm{PHGH}$ concentration of $0.039 \mathrm{~mol} / \mathrm{L}$ and initiator concentration of $4 \mathrm{mmol} / \mathrm{L}$, by which the maximum grafting ratio and efficiency of $18.45 \%$ and $58.45 \%$ were reached, respectively. Furthermore, the mechanical properties of the paper sheets with addition of CX-g-PHGH had obvious improvements. Compared to the mechanical properties of paper sheet without any addition of the xylan derivatives, the addition of CX-g-PHGH improved the mechanical properties of the sheets by up to $20.07 \%$ (tensile index), $25.31 \%$ (tear index), $30.20 \%$ (burst index) and $77.78 \%$ (folding endurance). Meanwhile, the paper sheet with addition of CX-g-PHGH exhibited improved viscosity, thermal stability as well as excellent antimicrobial activity against $E$. Coli bacterial which was inherited from the antimicrobial activity of guanidine in CX-g-PHGH.

The present work found a new way of synthesizing xylan-derivative and used it as antimicrobial additive agent against $E$. Coli bacteria in paper product. The obtained paper product with highly improved mechanical strength, antimicrobial property as well as biodegradable and renewable properties would find great potential applications especially in food-related areas, e.g. packaging, baking and napkin paper, as well as other areas such as pharmaceuticals, cosmetics and so on, which expanded the 
564 565

566

567

568

569

570

571

572

applications of xylan in more high value-added areas.

Author Contributions: Guibin $\mathrm{Xu}$, Bei $\mathrm{He}$ and Minmin Chang performed the laboratory work; Guibin Xu wrote the main manuscript text; Tao Song and Junli Ren supervised and revised the manuscript.

Funding: This work was financially supported by State Key Laboratory of Pulp and Paper Engineering, grants number 2017C02 and 201714, Science and Technology Planning Project of Guangdong Province, grant number 2017A010103032, The Guangdong Program for Support of Top-notch Young Professionals, grant number 2016TQ03Z585, the Fundamental Research Funds for the Central Universities of SCUT, grants number 2017ZD081 and 2017MS080, Guangdong Provincial Natural Science Foundation Project, grant number 2018A030313211 and Guangdong Province Science Foundation for Cultivating National Engineering Research Center for Efficient Utilization of Plant Fibers, grant number 2017B090903003.

Conflicts of Interest: The authors declare no conflict of interest.

\section{References}

1. Yang, R.C.; Qu, X.Y.; Xiao, S.Y.; Li, L.; Xu, B.J.; Fu, J.Y.; Lv, Y.J.; Amjad, N.; Tan, C.; Kim, K.S.; Chen, H.C.; Wang, X.R. Meningitic Escherichia coli-induced upregulation of PDGF-B and ICAM-1 aggravates blood-brain barrier disruption and neuroinflammatory response. J. Neuroinflamm. 2019, 16, 101-116. 
586

587

588

589

590

591

592

593

594

595

596

597

598

599

600

601

602

603

604

605

606

607

2. Kim, K.S. Pathogenesis of bacterial meningitis: From bacteraemia to neuronal injury. Nat. Rev. Neurosci. 2003, 4, 376-385.

3. Conrad, C.C.; Stanford, K.; McAllister, T.A.; Thomas, J.; Reuter, T. Further development of sample preparation and detection methods for $\mathrm{O} 157$ and the top 6 non-O157 STEC serogroups in cattle feces. J. Microbiol. Methods. 2014, 105, 2230.

4. Wang, X.; Maruvada, R.; Morris, A.J.; Liu, J.O.; Wolfgang, M.J.; Baek, D.J.; Bittman, R.; Kim, K.S. Sphingosine 1-phosphate activation of EGFR as a novel target for meningitic Escherichia coli penetration of the blood-brain barrier. Plos. Pathog. 2016, 12, e1005926.

5. Karmali, M.A. Emerging public health challenges of Shiga toxin-producing Escherichia coli related to changes in the pathogen, the population, and the environment. Clin. Infect. Dis. 2017, 64, 371-376.

6. Liu, W.T.; Lv, Y.J.; Yang, R.C.; Fu, J.Y.; Liu, L.; Wang, H.; Cao, Q.; Tan, C.; Chen, H.C.; Wang, X.R. New insights into meningitic Escherichia coli infection of brain microvascular endothelial cells from quantitative proteomics analysis. $J$. Neuroinflamm. 2018, 15, 291-310.

7. Manage, D.P.; Lauzon, J.; Jones, C.M.; Ward, P.J.; Pilarski, L.M.; Pilarski, P.M.; McMullen, L.M. Detection of pathogenic Escherichia coli on potentially contaminated beef carcasses using cassette PCR and conventional PCR. BMC Microbiologi. 2019, 19, 175-186.

8. Lin, L.; Agyemang, K.; Abdel-Samie, M.A.; Cui, H.Y. Antibacterial mechanism of 
608

609

610

611

612

613

614

615

616

617

618

619

620

621

622

623

624

625

626

627

628

629

Tetrapleura tetraptera extract against Escherichia coli and Staphylococcus aureus and its application in pork. J. Food. Safety. 2019, e12693

9. Poverenov, E.; Shemesh, M.; Gulino, A.; Cristaldi, D.A.; Zakin, V.; Yefremov, T.;

Granit, R. Durable contact active antimicrobial materials formed by a one-step covalent modification of polyvinyl alcohol, cellulose and glass surfaces. Colloids. Surf. B: Biointerfaces. 2013, 112, 356-361.

10. Shaer, A.E.; Hanson, P.; Worthington, T.; Lambert, P.; Mohammed, A.R. Preparation and characterization of amino acids-based trimethoprim salts. Pharmaceutics 2012, 4, 179-196.

11. Demetrescu, I.; Dumitriu, C.; Totea, G.; Nica, C.; Dinischiotu, A.; Ionita, D. Zwitterionic cysteine drug coating influence in functionalization of implantable Ti50Zr alloy for antibacterial, biocompatibility and stability properties. Pharmaceutics 2018, 10, 220.

12. Diaz-García, D.; Ardiles, P.R.; Prashar, S.; Rodriguez-Diéguez, A.; Páez, P.L.; Gómez-Ruiz, S. Preparation and study of the antibacterial applications and oxidative Stress induction of copper maleamate-functionalized mesoporous silica nanoparticles. Pharmaceutics 2019, 11, 30.

13. Dutta, P.K.; Tripathi, S.; Mehrotra, G.K.; Dutta, J. Perspectives for chitosan based antimicrobial films in food applications. Food. Chem. 2009, 114, 1173-1182.

14. Kang, Z.Z.; Zhang, B.; Jiao, Y.C.; Xu, Y.H.; He, Q.Z. High-efficacy antimicrobial cellulose grafted by a novel quaternarized N-halamine. Cellulose 2013, 20, 885-893.

15. Guan, Y.; Qian, L.Y.; Xiao, H.N.; Zheng, A. Preparation of novel antimicrobial- 
630

631

632

633

634

635

636

637

638

639

640

641

642

643

644

645

646

647

648

649

650

651

modified starch and its adsorption on cellulose fibers: Part I. Optimization of synthetic conditions and antimicrobial activities. Cellulose 2008, 15, 609-618.

16. Wu, J.; Zheng, Y.D.; Song, W.H.; Luan, J.B.; Wen, X.X.; Wu, Z.G.; Chen, X.H.; Wang, Q.; Guo, S.L. In situ synthesis of silver-nanoparticles/bacterial cellulose composites for slow-released antimicrobial wound dressing. Carbohyd. Polym. 2014, $102,762-771$.

17. Abreu, A.S.; Oliveira, M.; Sá, A.D.; Rui, M.R.; Cerqueira, M.A.; Vicente, A.A.; Machado, A.V. Antimicrobial nanostructured starch based films for packaging. Carbohyd. Polym. 2015, 129, 127-134.

18. Qian, L.Y.; Guan, Y.; He, B.H.; Xiao, H.N. Synergy of wet strength and antimicrobial activity of cellulose paper induced by a novel polymer complex. Mater. Lett. 2008, 62, 3610-3612.

19. Qian, L.Y.; Xiao, H.N.; Zhao, G.L.; He, B.H. Synthesis of modified guanidinebased polymers and their antimicrobial activities revealed by AFM and CLSM. ACS Appl. Mater. Inter. 2011, 3, 1895-1901.

20. Qian, L.Y.; Li, X.; Sun, S.L.; Xiao, H.N. Preparation of guanidine polymer and its complex as dual-functional agent for cellulose fibre-based hygiene products. $J$. Biobased. Mater. Bio. 2011, 5, 219-224.

21. Sun, S.L.; An, Q.Z.; Li, X.; Qian, L.Y.; He, B.H.; Xiao, H.N. Synergistic effects of chitosan-guanidine complexes on enhancing antimicrobial activity and wet-strength of paper. Bioresource. Technol. 2010, 101, 5693-5700.

22. Ebringerová, A.; Heinze, T. Xylan and xylan derivatives-biopolymers with 
652

653

654

655

656

657

658

659

660

661

662

663

664

665

666

667

668

669

670

671

672

673

valuable properties. I. Naturally occurring xylans structures, isolation procedures and properties. Macromol. Rapid. Comm. 2015, 21, 542-556.

23. Zyl, C.V.; Prior, B.A.; Preez, J.C.D. Production of ethanol from sugar cane bagasse hemicellulose hydrolyzate by Pichia stipitis. Appl. Biochem. Biotech. 1988, 17, 357369.

24. Sun, R.C. Cereal straw as a resource for sustainable biomaterials and biofuels. Elsevier, The Netherlands Linacre House, Jordan Hill, Oxford, UK. 2010.

25. Horton, D. Advances in Carbohydrate Chemistry: Volume 21: M.L. Wolfrom and R. S. Tipson. Academic Press Inc., New York. 1968.

26. Hromádková, Z.; Kováčiková, J.; Ebringerová, A. Study of the classical and ultrasound-assisted extraction of the corn cob xylan. Ind. Crops. Products. 1999, 9, 101-109.

27. Ünlü, C.H.; Günister, E.; Atıc1, O. Synthesis and characterization of NaMt biocomposites with corn cob xylan in aqueous media. Carbohyd. Polym. 2009, 76, $585-592$.

28. Li, X.X.; Shi, X.W.; Wang, M.; Du, Y.M. Xylan chitosan conjugate-a potential food preservative. Food. Chem. 2011, 126, 520-525.

29. Petzold, K.; Schwikal, K.; Heinze, T. Carboxymethyl xylan-synthesis and detailed structure characterization. Carbohyd. Polym. 2006, 64, 292-298.

30. Ren, J.L.; Sun, R.C.; Liu, C.F.; Cao, Z.N.; Luo, W. Acetylation of wheat straw hemicelluloses in ionic liquid using iodine as a catalyst. Carbohyd. Polym. 2007, 70, 406-414. 
674

675

676

677

678

679

680

681

682

683

684

685

686

687

688

689

690

691

692

693

694

695

31. Ren, J.L.; Xu, F.; Sun, R.C.; Peng, B.; Sun, J.X. Studies of the lauroylation of wheat straw hemicelluloses under heating. J. Agric. Food Chem. 2008, 56, 1251-1258.

32. Schwikal, K.; Heinze, T.; Ebringerová, A.; Petzold, K. Cationic xylan derivatives with high degree of functionalization. Macromol. Symp. 2010, 232, 49-56.

33. Goksu, E.I.; Karamanlioglu, M.; Bakir, U.; Yilmaz, L.; Yilmazer, U. Production and characterization of films from cotton stalk xylan. J. Agric. Food Chem. 2007, $55,10685-10691$.

34. Hansen, N.M.L.; Plackett, D. Sustainable films and additives from hemicelluloses: a review. Biomacromolecules 2008, 9, 1493-1505.

35. Lindblad, M.S.; Ranucci, E.; Albertsson, A.C. Biodegradable polymers from renewable sources. new hemicellulose - based hydrogels. Macromol. Rapid. Comm. 2001, 22, 962-967.

36. Melosilveira, R.F.; Fidelis, G.P.; Telles, C.B.S.; Dantassantos, N.; Elias, S.D.O.; Ribeiro, V.B.; Barth, A.L.; Macedo, A.J.; Leite, E.L. In vitro antioxidant, anticoagulant and antimicrobial activity and in inhibition of cancer cell proliferation by xylan extracted from corn cobs. Int. J. Mol. Sci. 2011, 13, 409-426.

37. Pristov, J.B.; Mitrović, A.; Spasojević, I. A comparative study of antioxidative activities of cell-wall polysaccharides. Carbohyd. Res. 2011, 346, 2255-2259.

38. Ren, J.L.; Wang, S.Y.; Gao, C.D.; Chen, X.F.; Li, W.Y.; Peng, F. TiO2-containing PVA/xylan composite films with enhanced mechanical properties, high hydrophobicity and UV shielding performance. Cellulose 2015, 22, 593-602.

39. Wang, X.Y.; Liu, B.; Ren, J.L.; Liu, C.F.; Wang, X.H.; Wu, J.; Sun, R.C. 
696

697

698

699

700

701

702

703

704

705

706

707

708

709

710

711

712

713

Preparation and characterization of new quaternized carboxymethyl chitosan/rectorite nanocomposite. Compos. Sci. Technol. 2010, 70, 1161-1167.

40. Bao, Y.P.; Zhang, H.; Luan, Q.; Zheng, M.M.; Tang, H.; Huang, F.H. Fabrication of cellulose nanowhiskers reinforced chitosan-xylan nanocomposite films with antibacterial and antioxidant activities. Carbohyd. Polym. 2018, 184, 66-73.

41. Queirós, L.C.C.; Sousa, S.C.L.; Duarte, A.F.S.; Domingues, F.C.; Ramos, A.M.M. Development of carboxymethyl xylan films with functional properties. J. Food. Sci. Technol. 2017, 54, 9-17.

42. Guan, Y.; Xiao, H.N.; Sullivan, H.; Zheng, A. Antimicrobial-modified sulfite pulps prepared by in situ copolymerization. Carbohyd. Polym. 2007, 69, 688-696.

43. Liu, K.; Xu, Y.G.; Lin, X.X.; Chen, L.H.; Huang, L.L.; Cao, S.L.; Li, J. Synergistic effects of guanidine-grafted CMC on enhancing antimicrobial activity and dry strength of paper. Carbohyd. Polym. 2014, 110, 382-387.

44. Peng, X.W.; Ren, J.L.; Zhong, L.X.; Sun, R.C. Synthesis and characterization of amphoteric xylan-type hemicelluloses by microwave irradiation. J. Agric. Food Chem. 2012, 60, 1695.

45. Peng, X.W.; Ren, J.L.; Sun, R.C. An efficient method for the synthesis of hemicellulosic derivatives with bifunctional groups in butanol/water medium and their rheological properties. Carbohyd. Polym. 2011, 83, 1922-1928.

46. Raj, S.B.; Kumar, V.; Soni, P.L. Ceric ammonium nitrate - initiated graft copolymerization of acrylamide onto Cassia tora gum. J. Appl. Polym. Sci. 2010, 86, $3250-3255$ 
718

719

720

721

722

723

724

725

726

727

47. Singh, V.; Tiwari, A.; Tripathi, D.N.; Sanghi, R. Microwave assisted synthesis of Guar-g-polyacrylamide. Carbohyd. Polym. 2004, 58, 1-6.

48. Wang, L.L.; Xu, Y.S. Preparation and characterization of graft copolymerization of ethyl acrylate onto hydroxypropyl methylcellulose in aqueous medium. Cellulose 2006, 13, 191-200.

49. Xu, G.B.; Kong, W.Q.; Liu, C.F.; Sun, R.C.; Ren, J.L. Synthesis and Characteristic of Xylan-grafted-polyacrylamide and Application for Improving Pulp Properties. Materials 2017, 10, 971-982.

50. Kong, W.Q.; Ren, J.L.; Wang, S.Y.; Li, M.F.; Sun, R.C. A promising strategy for preparation of cationic xylan by environment-friendly semi-dry oven process. Fiber. Polym. 2014, 15, 943-949.

51. Katsura, S.; Isogai, A.; Onabe, F.; Usuda, M. NMR analyses of polysaccharide derivatives containing amine groups. Carbohyd. Polym. 1992, 18, 283-288.

52. Tanodekaew, S.; Channasanon, S. Xylan/polyvinyl alcohol blend and its performance as hydrogel. J. Appl. Polym. Sci. 2010, 100, 1914-1918.

53. Eutamene, M.; Benbakhti, A.; Khodja, M.; Jada, A. Preparation and Aqueous

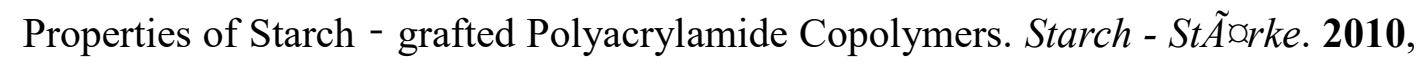
$61,81-91$

54. Yoo, D.; Kim, C.; Yoo, B. Steady and Dynamic Shear Rheology of Rice Starch Galactomannan Mixtures. Starch-Starke. 2005, 57, 310-318.

55. Maschio, G.; Koufopanos, C.; Lucchesi, A. Pyrolysis, a promising route for biomass utilization. Bioresource. Technol. 1992, 42, 219-231. 
740 56. Ren, J.L.; Peng, F.; Sun, R.C.; Kennedy, J.F. Influence of hemicellulosic 741 derivatives on the sulfate kraft pulp strength. Carbohyd. Polym. 2009, 75, 338-342.

742 57. Lima, D.U.; Oliveira, R.C.; Buckeridge, M.S. Seed storage hemicelluloses as wet743 end additives in papermaking. Carbohyd. Polym. 2003, 52, 367-373. 